\title{
Tunneling Through a One-Dimensional Square Potential Barrier Under Fluctuations in an Observer's Frame of Reference
}

\author{
Yun-Sok Shin \\ Sejong Academy of Science and Arts, Sejong, Republic of Korea
}

\section{Email address:}

yunsokshin@naver.com

\section{To cite this article:}

Yun-Sok Shin. Tunneling Through a One-Dimensional Square Potential Barrier Under Fluctuations in an Observer's Frame of Reference. American Journal of Physics and Applications. Vol. 8, No. 3, 2020, pp. 40-45. doi: 10.11648/j.ajpa.20200803.12

Received: May 11, 2020; Accepted: June 2, 2020; Published: June 15, 2020

\begin{abstract}
This study reports tunneling through a one-dimensional (1D) square potential barrier (SPB) under fluctuations in an observer's frame of reference (OFR). To date, tunneling through an SPB has been studied under the assumption that the OFR remains constant throughout the tunneling measurements; therefore, the change of the tunneling probability when the OFR is assumed to fluctuate remains unanswered. In this paper, a 1D SPB is considered under fluctuations of an OFR. The average transmission probability of a particle through an SBP for two types of OFR fluctuations (periodic-square-wave and periodic-sawtooth-wave fluctuations) is formulated in time representations. Under these types of fluctuations, the average transmission probability gradually increases with a particle's energy, which is saturated to the transmission probability in the case of the stationary OFR at a much greater energy than the amplitude of the fluctuations. The average transmission probability is much higher at the amplitude of the fluctuations in the case of periodic-square-wave fluctuations. Therefore, the average transmission probability with a particle's energy has the potential to reveal the distribution of OFR fluctuations.
\end{abstract}

Keywords: Tunneling, Potential Barrier, Observer Effect, Fluctuations of an Observer's Frame of Reference, Fluctuating Frame of Reference

\section{Introduction}

Tunneling is the quantum mechanical phenomenon in which a particle passes through a potential barrier. It is fundamental to understanding the wave nature of particles [1$3]$ and plays a central role in various practical applications, such as in radioactive disintegration [4-11], electron tunneling devices [12-15], quantum computation [16-22], and scanning tunneling microscopy [23-29].

Particle tunneling can occur in a potential barrier with a thickness in the order of nanometers or less [23-25]. Tunneling can be estimated in terms of Heisenberg's uncertainty principle [3], which holds that measuring the momentum (position) of a particle can be conducted with precision, whereas the resulting position (momentum) disturbance of the particle is limited by Planck's constant divided by the uncertainty of the momentum (position) of the particle. When a particle is located at a potential barrier, it can pass through the barrier. This is because a particle has uncertainty in terms of its momentum; additionally, according to Heisenberg's uncertainty principle, the resulting position disturbance of the particle can be greater than the width of the barrier. For an example, a square potential barrier (SPB) provides an exact solution for calculating the transmission probability of a particle; it can quantitatively calculate tunneling as well as provide a realistic approximation of particle tunneling.

In this paper, particle tunneling was quantitatively studied according to the transmission probability of a particle through a potential barrier. To date, tunneling has been examined under the assumption that an observer's frame of reference (OFR) remains constant. In much of the research, the OFR is assumed to be zero throughout the tunneling measurements. Therefore, the change of the tunneling probability when the OFR is assumed to fluctuate currently remains unanswered.

Recently, a novel observer effect induced by OFR fluctuations was proposed in an Einstein solid [30] and a 
single-electron transistor (SET) [31]. The molar specific heat at a constant volume of an Einstein solid as a function of temperature revealed the distribution of OFR fluctuations, which exhibited a peak and convergence of three times the level of the gas constant at low temperatures under periodicsquare-wave and periodic-sawtooth-wave fluctuations, respectively. Regarding the SET, the average current in an SET can also reveal the distribution of OFR fluctuations. An SET comprised a source, drain, and single channel. The average current in it exhibited an asymmetric zero-bias Coulomb peak as a function of the energy of the channel under periodic-square-wave and periodic-sawtooth-wave fluctuations - the amplitude of which gradually increased as the amplitude of the fluctuations increased. The amplitude of the zero-bias Coulomb peak was greater in the case of periodic-square-wave fluctuations.

An observer effect induced by fluctuations of the OFR can be investigated if the reference of energy of a particle is matched to an OFR. In this study, the average transmission probability of a particle to transmit an SPB [32] was investigated under OFR fluctuations. The average transmission probability was formulated for two types of fluctuations in time representations, namely, periodic-squarewave fluctuation and periodic-sawtooth-wave fluctuation. Under these types of fluctuations, the average transmission probability monotonically increases with the energy of the particle, which is saturated to the transmission probability in the case of a stationary OFR at a much greater energy than the amplitude of the fluctuations. The average transmission probability rapidly increases just above the energy corresponding to the amplitude of fluctuations of the OFR in the case of periodic-square-wave fluctuations. Therefore, the average transmission probability with a particle's energy may be able to reveal the distribution of OFR fluctuations.

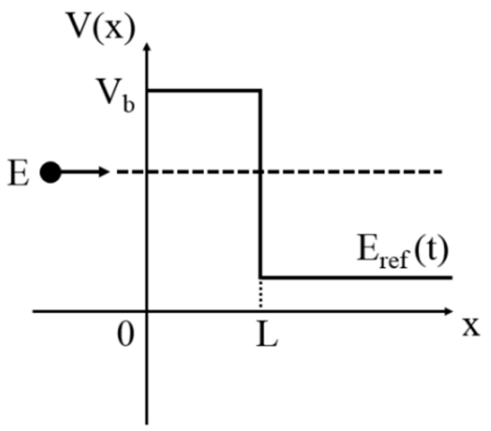

Figure 1. Schematic of a $1 D S P B$, where $V_{b}$ and $L$ are potential energy and width of the barrier, respectively, $E$ the energy of a particle, $E_{r e f}(t)$ is the energy of an OFR at time $t$. The transmission probability through the barrier was monitored by the observer on the right side of the barrier.

\section{Average Transmission Probability Through a One-Dimensional Square Potential Barrier}

Figure 1 shows a schematic of a one-dimensional (1D) SPB, where $V_{\mathrm{b}}$ and $L$ are the potential energy and width of the barrier, respectively, $E$ the energy of a particle, and $E_{\mathrm{ref}}(t)$ the energy of an OFR at time $t$. In this study, $E_{\mathrm{ref}}(t)$ was assumed to be constant at a time interval between $t$ and $t+d t$, where $\mathrm{d} t \ll \Delta \mathrm{t}\left(=t_{f}-t_{i}\right)$. Here, $t_{i}$ and $t_{f}$ are the initial and final times, respectively. If a particle from left (at $\mathrm{x} \ll 0$ ) was located at the 1D SPB, then the particle's transmission through the barrier was monitored by the observer far from the 1D SPB (at $\mathrm{x} \gg L$ ).

In terms of the energy of a particle, $E \geq V_{\mathrm{b}}$, the wave function of a particle under the $1 \mathrm{D}$ SPB at $t, \Psi(x, t)=$ $\varphi(x) e^{-i E t / \hbar}$, where $\varphi(x)$ is expressed as

$$
\varphi(x)=\left\{\begin{array}{c}
e^{i k x}+R e^{-i k x} \text { for }-\infty<x<0 \\
A e^{i \alpha x}+B e^{-i \alpha x} \text { for } 0 \leq x<L, \\
T e^{i q x} \text { for } L \leq x<\infty
\end{array}\right.
$$

where $\quad k=\sqrt{2 m E} / \hbar \quad, \quad \alpha=\sqrt{2 m\left(E-V_{b}\right)} / \hbar, \quad q=$ $\sqrt{2 m E(t)} / \hbar$. Here, $m$ is the mass of the particle, $\hbar$ the Planck's constant divided by $2 \pi, E(t)=E-E_{\text {ref }}(t)$ the measured energy for $E$ at time $t$, and $E \geq E_{\text {ref }}(t) . R$ and $T$ is the coefficients of reflection and transmission of the particle, respectively. The transmission probability of the particle through the 1D SPB, $1-|R|^{2}$, can be expressed as

$$
1-|R|^{2}=\frac{8 \alpha^{2} k q}{\left(\alpha^{2}+k^{2}\right)\left(\alpha^{2}+q^{2}\right)+4 \alpha^{2} k q-\left(\alpha^{2}-k^{2}\right)\left(\alpha^{2}-q^{2}\right) \cos (2 \alpha L)},
$$

In terms of the energy of the particle, $0 \leq E<V_{\mathrm{b}}$, the wave function of the particle under the $1 \mathrm{D} \mathrm{SPB}$ at $t$, $\Psi(x, t)=\varphi(x) e^{-i E t / \hbar}$, where $\varphi(x)$ is expressed as

$$
\varphi(x)=\left\{\begin{array}{c}
e^{i k x}+R e^{-i k x} \text { for }-\infty<x<0 \\
A e^{-\beta x}+B e^{\beta x} \text { for } 0 \leq x<L, \\
T e^{i q x} \text { for } L \leq x<\infty
\end{array},\right.
$$

where $\beta=\sqrt{2 m\left(V_{b}-E\right)} / \hbar$. The transmission probability of the particle through the 1D SPB, $1-|R|^{2}$, can be expressed as

$$
1-|R|^{2}=\frac{8 \beta^{2} k q}{-\left(\beta^{2}-k^{2}\right)\left(\beta^{2}-q^{2}\right)+4 \beta^{2} k q+\left(\beta^{2}+k^{2}\right)\left(\beta^{2}+q^{2}\right) \cosh (2 \beta L)},
$$

For the energy of an OFR at time $t, E_{\text {ref }}(t) \leq E<V_{\mathrm{b}}$, the average transmission probability through the 1D SPB with time interval $\Delta \mathrm{t}, 1-\left|R_{\mathrm{avg}}\right|^{2}$, can be expressed as

$$
1-\left|R_{\mathrm{avg}}\right|^{2}=\frac{1}{\Delta t} \int_{t_{i}}^{t_{f}} \frac{8 \beta^{2} k q}{-\left(\beta^{2}-k^{2}\right)\left(\beta^{2}-q^{2}\right)+4 \beta^{2} k q+\left(\beta^{2}+k^{2}\right)\left(\beta^{2}+q^{2}\right) \cosh (2 \beta L)} d t
$$

For an observer with a stationary frame of reference ( $E_{\mathrm{ref}}(t)=0$ ), the corresponding transmission probability, $1-\left|R_{0}\right|^{2}$, can be expressed as

$$
1-\left|R_{0}\right|^{2}=\frac{4 E\left(V_{b}-E\right)}{4 E\left(V_{b}-E\right)+V_{b}^{2} \sinh ^{2}\left(L \sqrt{2 m\left(V_{b}-E\right)} / \hbar\right)} .
$$

It has been found that the transmission probability of a particle in stationary OFR, $1-\left|R_{0}\right|^{2}$, is 0 at $E=0$ and then 
gradually increases with increasing $E$. In the limit of $\beta L \ll 1$, $1-\left|R_{0}\right|^{2} \approx 1$ (i.e., the barrier is sufficiently thin to be transparent). In the limit of $\beta L \gg 1,1-\left|R_{0}\right|^{2} \approx\left\{16 \mathrm{E}\left(V_{b}-\right.\right.$ $\left.E) / V_{b}^{2}\right\} \exp \left(-2 L \sqrt{2 m\left(V_{b}-E\right)} / \hbar\right)$ (i.e., overall $1-\left|R_{0}\right|^{2}$ exponentially increases with increasing $E$ ). (a)

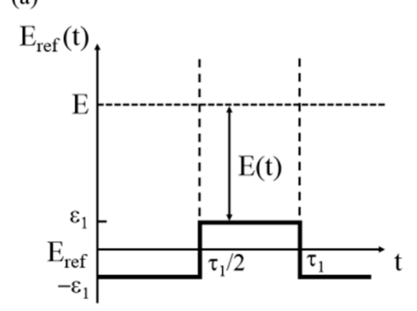

(b)

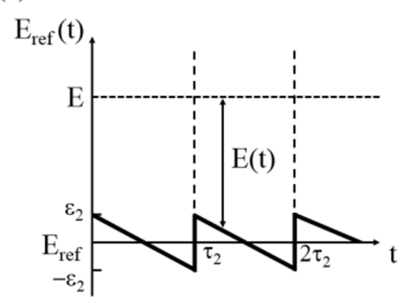

Figure 2. (a) Fluctuating frame of reference at time $t, E_{\text {ref }}(t)$, for periodic-square-wave fluctuations, of which the amplitude and period are $\varepsilon_{1}$ and $\tau_{1}$, respectively. Measured energy for $E$ at time $t, E(t)(=E-$ $\left.E_{\text {ref }}(t)\right)$, and stationary frame of reference, $E_{\text {ref }}$, which was considered to be equal to 0 . (b) Fluctuating frame of reference at time $t, E_{\text {ref }}(t)$, for periodic-sawtooth-wave fluctuations, of which the amplitude and period are $\varepsilon_{2}$ and $\tau_{2}$, respectively.

\section{Average Transmission Probability Through a 1D SPB Under Periodic- Square-Wave Fluctuations}

Given periodic $\mathrm{E}_{\text {ref }}(t)=\left\{\begin{array}{l}-\varepsilon_{1} \text { for } 0 \leq t<\tau_{1} / 2 \\ \varepsilon_{1} \text { for } \tau_{1} / 2 \leq t<\tau_{1}\end{array}\right.$ (figure 2(a)), the measured energy for $E$ at time $t, E(t)$, can be expressed as

$$
E(t)= \begin{cases}E+\varepsilon_{1} & \text { for } 0 \leq t<\tau_{1} / 2 \\ E-\varepsilon_{1} & \text { for } \tau_{1} / 2 \leq t<\tau_{1}\end{cases}
$$

where $\varepsilon_{1}$ and $\tau_{1}$ are the amplitude and period of the periodic-square-wave fluctuations, respectively.

\subsection{Periodic-Square-Wave Fluctuations of a Half Period}

For an observer with a fluctuating frame of reference by means of half-period periodic-square-wave fluctuations, the corresponding transmission probability, $1-\left|R_{1, \mathrm{j}}\right|^{2}$, is expressed as

$$
1-\left|R_{1,1}\right|^{2}=\frac{8\left(V_{b}-E\right) \sqrt{E\left(E+\varepsilon_{1}\right)}}{-\left(V_{b}-2 E\right)\left(V_{b}-2 E-\varepsilon_{1}\right)+4\left(V_{b}-E\right) \sqrt{E\left(E+\varepsilon_{1}\right)}+V_{b}\left(V_{b}+\varepsilon_{1}\right) \cosh \left(2 L \sqrt{2 m\left(V_{b}-E\right)} / \hbar\right)},
$$

where $t_{i}=0$ and $t_{f}=\tau_{1} / 2$. Here, $\mathrm{j}=1$.

$$
1-\left|R_{1,2}\right|^{2}=\frac{8\left(V_{b}-E\right) \sqrt{E\left(E-\varepsilon_{1}\right)}}{-\left(V_{b}-2 E\right)\left(V_{b}-2 E+\varepsilon_{1}\right)+4\left(V_{b}-E\right) \sqrt{E\left(E-\varepsilon_{1}\right)}+V_{b}\left(V_{b}-\varepsilon_{1}\right) \cosh \left(2 L \sqrt{2 m\left(V_{b}-E\right)} / \hbar\right)},
$$

where $t_{i}=\tau_{1} / 2$ and $t_{f}=\tau_{1}$. Here, $\mathrm{j}=2$.

\subsection{Periodic-Square-Wave Fluctuations of One Period}

For an observer with a fluctuating frame of reference by means of one-period periodic-square-wave fluctuations, the corresponding transmission probability, $1-\left|R_{1}\right|^{2}$, is expressed as

$$
1-\left|R_{1}\right|^{2}=\left\{\begin{array}{c}
0.5\left(1-\left|R_{1,1}\right|^{2}\right)+0.5\left(1-\left|R_{1,2}\right|^{2}\right) \text { for } E \geq \varepsilon_{1} \\
0.5\left(1-\left|R_{1,1}\right|^{2}\right) \text { for } 0 \leq E<\varepsilon_{1}
\end{array}\right.
$$

where $t_{i}=0$ and $t_{f}=\tau_{1}$.

As shown in figure 3 , the average transmission probability

(a)

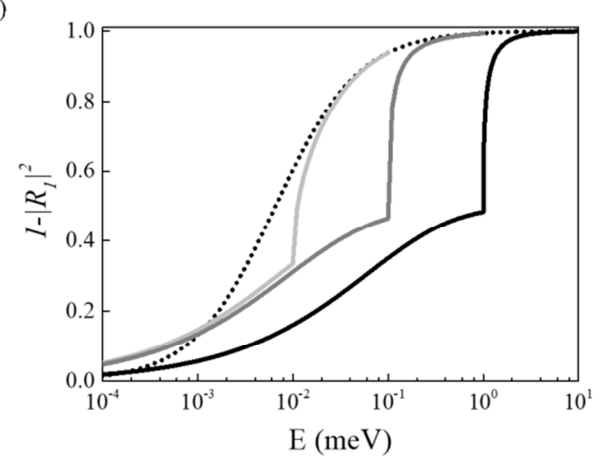

of a particle with an electron's mass, $1-\left|R_{1}\right|^{2}$, is displayed as a function of $E$, where $V_{\mathrm{b}}=1 \mathrm{eV}$ [33]. $1-\left|R_{1}\right|^{2}$ is 0 at $E=0$ and then gradually increases with $E$ to just above $E=\varepsilon_{1}$ and is saturated to the transmission probability in the case of the stationary $\operatorname{OFR}\left(\varepsilon_{1}=0\right), 1-\left|R_{0}\right|^{2}$. In the limit of $\beta L \ll 1$ and $\varepsilon_{1} \ll E<V_{\mathrm{b}}, 1-\left|R_{1}\right|^{2} \approx 1$. As an example, for a SPB with $L=1 \mathrm{pm}$, the barrier is transparent at high energies, as shown in figure 3(a). In the limit of $\beta L \gg 1$ and $E \ll \varepsilon_{1}, \quad 1-\left|R_{1}\right|^{2} \approx\left\{8\left(V_{b}-E\right) \sqrt{E \varepsilon_{1}} /\right.$ $\left.V_{b}^{2}\right\} \exp \left(-2 L \sqrt{2 m\left(V_{b}-E\right)} / \mathrm{h}\right)$. For a SPB with $L=1 \mathrm{~nm}$, overall $1-\left|R_{1}\right|^{2}$ exponentially increases with increasing $E$ and is distinguished from $1-\left|R_{0}\right|^{2}$, as shown in figure 3(b).

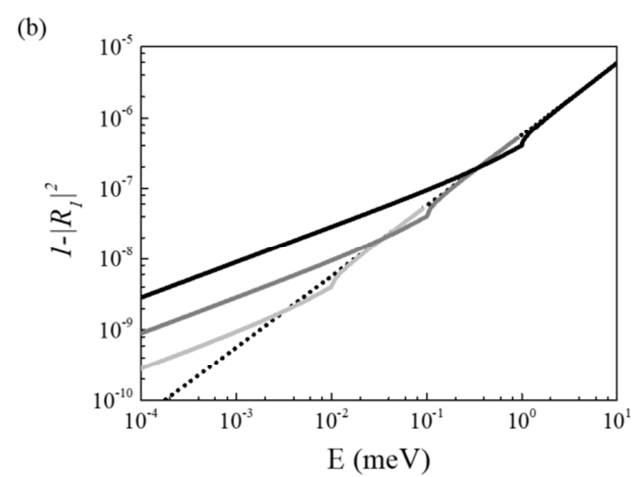

Figure 3. Average transmission probability of a particle with an electron's mass through the $1 D$ SPB under the periodic-square-wave fluctuations, 1 - $\left|R_{1}\right|^{2}$, as a function of $E$ at (a) $L=1 \mathrm{pm}$ and (b) $L=1 \mathrm{~nm}$. Here, $\varepsilon_{1}=0.01 \mathrm{meV}$ (light gray), $0.1 \mathrm{meV}$ (gray), and $1 \mathrm{meV}$ (black). The dotted lines denote the transmission probability of the particle under stationary OFR, $1-\left|R_{0}\right|^{2}$, which corresponds to $1-\left|R_{1}\right|^{2}$ at $\varepsilon_{1}=0$. $V_{b}$ is set as 1 eV. 


\subsection{Periodic-Square-Wave Fluctuations at High-Frequency Limits}

For an observer with a fluctuating frame of reference by means of periodic-square-wave fluctuations at highfrequency limits, the corresponding transmission probability, $1-\left|R_{1}{ }^{\prime}\right|^{2}$, is expressed as

$$
1-\left|R_{1}^{\prime}\right|^{2} \approx 1-\left|R_{1}\right|^{2}
$$

where $t_{i}=0$ and $t_{f} \gg \tau_{1}$.

\section{Average Transmission Probability Through a 1D SPB Under Periodic- Sawtooth-Wave Fluctuations}

Given periodic $\mathrm{E}_{r e f}(t)=\varepsilon_{2}-\frac{2 \varepsilon_{2}}{\tau_{2}} t$ for $0 \leq t<\tau_{2}$ (figure 2(b)), the measured energy for $E$ at time $t, E(t)$, where

$$
1-\left|R_{2,1}\right|^{2}=\frac{1}{2 \varepsilon_{2}} \int_{-\varepsilon_{2}}^{\varepsilon_{2}} \frac{8\left(V_{b}-E\right) \sqrt{E(E+\varepsilon)}}{-\left(V_{b}-2 E\right)\left(V_{b}-2 E-\varepsilon\right)+4\left(V_{b}-E\right) \sqrt{E(E+\varepsilon)}+V_{b}\left(V_{b}+\varepsilon\right) \cosh \left(2 L \sqrt{2 m\left(V_{b}-E\right)} / \mathrm{h}\right)} d \varepsilon,
$$

and

$$
1-\left|R_{2,2}\right|^{2}=\frac{1}{2 \varepsilon_{2}} \int_{-\varepsilon_{2}}^{E} \frac{8\left(V_{b}-E\right) \sqrt{E(E-\varepsilon)}}{-\left(V_{b}-2 E\right)\left(V_{b}-2 E+\varepsilon\right)+4\left(V_{b}-E\right) \sqrt{E(E-\varepsilon)}+V_{b}\left(V_{b}-\varepsilon\right) \cosh \left(2 L \sqrt{2 m\left(V_{b}-E\right)} / \mathrm{h}\right)} d \varepsilon .
$$

As shown in figure 4 , the average transmission probability of a particle with an electron's mass, $1-\left|R_{2}\right|^{2}$, is displayed as a function of $E$ where $V_{\mathrm{b}}=1 \mathrm{eV}[33] .1-\left|R_{2}\right|^{2}$ is 0 at $E=0$; it gradually increases with $E$ and is saturated to the transmission probability in the case of the stationary OFR, $1-\left|R_{0}\right|^{2}$. The change of $1-\left|R_{2}\right|^{2}$ with respect to $E$ around $\varepsilon_{2}$ is much slower than in the case of the periodicsquare-wave fluctuations, $1-\left|R_{1}\right|^{2}$. In the limit of $\beta L \ll 1$ and $\varepsilon_{2} \ll E<V_{\mathrm{b}}, 1-\left|R_{2}\right|^{2} \approx 1$ (i.e., the barrier is sufficiently thin so as to be transparent). For an SPB with $L=1 \mathrm{pm}$ as an extremely thin barrier, $1-\left|R_{2}\right|^{2}$ is saturated to 1 at high energies, as shown in figure 4(a). Figure 4(b) shows $1-\left|R_{2}\right|^{2}$ as a function of $E$ for a thicker SPB with $L=1 \mathrm{~nm}$, where the overall $1-\left|R_{2}\right|^{2}$ also exponentially increases with increasing $E$. In the limit of

(a)

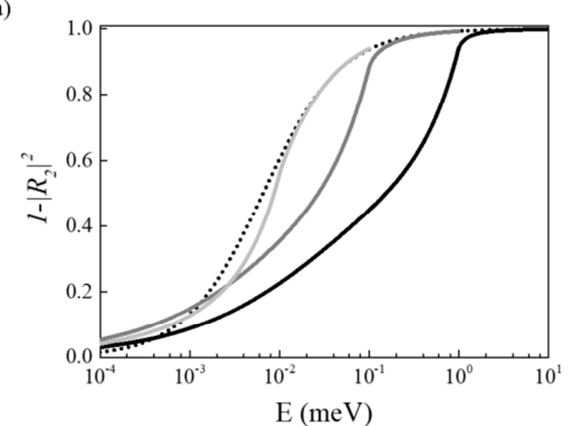

$\beta L \gg 1$ and $E \ll \varepsilon_{2}, \quad 1-\left|R_{2}\right|^{2} \approx\left\{16\left(V_{b}-E\right) \sqrt{E \varepsilon_{2}} /\right.$ $\left.3 V_{b}^{2}\right\} \exp \left(-2 L \sqrt{2 m\left(V_{b}-E\right)} / \mathrm{h}\right)$, which is clearly distinguished from both $1-\left|R_{0}\right|^{2}$ and $1-\left|R_{1}\right|^{2}$.

\subsection{Periodic-Sawtooth-Wave Fluctuations at High- Frequency Limits}

For an observer with a fluctuating frame of reference by means of periodic-sawtooth-wave fluctuations at highfrequency limits, the corresponding transmission probability, $1-\left|R_{2}^{\prime}\right|^{2}$, is expressed as

$$
1-\left|R_{2}^{\prime}\right|^{2} \approx 1-\left|R_{2}\right|^{2}
$$

where $t_{i}=0$ and $t_{f} \gg \tau_{2}$.

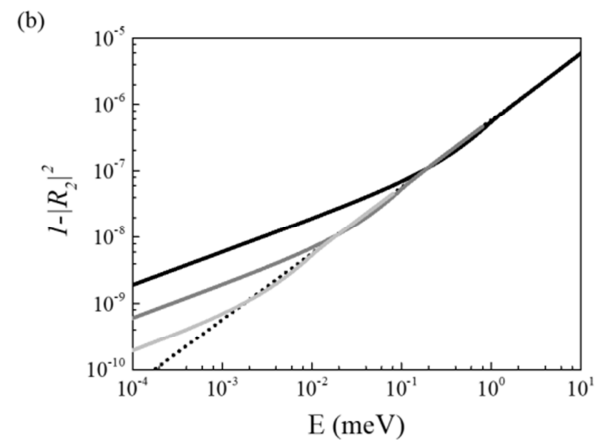

Figure 4. Average transmission probability of a particle with an electron's mass through the $1 D$ SPB under the periodic-sawtooth-wave fluctuations, 1 $\left|R_{2}\right|^{2}$, as a function of $E$ at (a) $L=1 \mathrm{pm}$ and (b) $L=1 \mathrm{~nm}$. Here, $\varepsilon_{2}=0.01 \mathrm{meV}$ (light gray), $0.1 \mathrm{meV}$ (gray), and $1 \mathrm{meV}$ (black). The dotted lines denote $1-\left|R_{0}\right|^{2}$. $V_{b}$ is set as $1 \mathrm{eV}$. 


\section{Conclusions}

In this paper, tunneling through an SPB was studied under the periodic fluctuations of an OFR. A particle's average transmission probability was quantitatively calculated. Furthermore, the average transmission probability was formulated for two types of fluctuations in time representations, namely, periodic-square-wave fluctuation and periodic-sawtooth-wave fluctuations, based on the assumption that the reference of the particle's energy was matched to the OFR. Under periodic-square-wave fluctuations of one period or at high-frequency limits, the average transmission probability was 0 at the energy of the particle, $E=0$; it gradually increased with the increasing energy of the particle, rapidly increased just above the amplitude of the fluctuations, and saturated to the transmission probability in the case of the stationary OFR. Similarly, under periodic-sawtooth-wave fluctuations of one period or at high-frequency limits, the average transmission probability gradually increased with the increasing energy of the particle but showed a much smoother increase above the amplitude of the fluctuations. Therefore, the average transmission probability through an SPB with a particle's energy provides clear criteria for characterizing the distribution of OFR fluctuations.

\section{Acknowledgements}

This work was supported by Sejong Academy of Science and Arts.

\section{References}

[1] L. de Broglie, "Recherches sur la théorie des quanta," Ann. Phys., vol. 10, 1925, pp. 22-128.

[2] E. Schrödinger, "An undulatory theory of the mechanics of atoms and molecules," Phys. Rev., vol. 28, 1926, pp. 10491070 .

[3] W. Heisenberg, "Über den anschaulichen Inhalt der quantentheoretischen Kinematik und Mechanik," Z. Phys., vol. 43, 1927, pp. 172-198.

[4] J. Elster and H. Geitel, "Bequerel rays," Wied. Ann., vol. 66, 1889 , p. 735.

[5] E. R. von Schweidler, Premier Congres de Radiologie, Liege, 1905.

[6] E. Rutherford, "Radioactive substance emitted from Thorium compounds," Phil. Mag., vol. 49, 1900, pp. 1-14.

[7] G. Gamow, "The quantum theory of atomic nucleus," Z. Phys., vol. 51, 1928, pp. 204-212.

[8] R. W. Gurney and E. U. Condon, "Wave mechanics and radioactive disintegration," Nature, vol. 122, 1928, p. 439.

[9] G. Gamow, "The quantum theory of nuclear disintegration," Nature, vol. 122, 1928, pp. 805-806.
[10] R. W. Gurney and E. U. Condon, "Quantum mechanics and radioactive disintegration," Phys. Rev., vol. 33, 1929, pp. 127 140.

[11] C. Détraz, "The discovery of radioactivity: A one-hundred year heritage," Nucl. Phys. A, vol. 654, 1999, pp. 12c-18c.

[12] R. H. Fowler and L. Nordheim, "Electric emission in intense electric fields," Proc. Roy. Soc. A, vol. 119, 1928, pp. 173-181.

[13] L. Esaki, "Long journey into tunneling," Proc. of the IEEE, vol. 62, 1974, pp. 825-831.

[14] I. Giaever, "Electron tunneling and superconductivity," Science, vol. 183, 1974, pp. 1253-1258.

[15] B. D. Josephson, "The discovery of tunneling supercurrent," Science, vol. 184, 1974, pp. 527-530.

[16] P. Benioff, "The computer as a physical system: A microscopic quantum mechanical Hamiltonian model of computers as represented by Turing machines," Journal of Statistical Physics, vol. 22,1980 , pp. 563-591.

[17] Y. I. Manin, "Computable and noncomputable (in Russian)," Sov. Radio., 1980, pp. 13-15.

[18] R. Feynman, "Simulating Physics with Computers," International Journal of Theoretical Physics, vol. 21, 1982, pp. 467-488.

[19] P. W. Shor, "Algorithms for quantum computation: discrete logarithms and factoring," Proceedings 35th Annual Symposium on Foundations of Computer Science, IEEE Comput. Soc., 1994, pp. 124-134.

[20] D. Loss and D. P. DiVincenzo, "Quantum computation with quantum dots," Phys. Rev. A, vol. 57, 1998, pp. 120-126.

[21] J. Clarke and F. K. Wilhelm, "Superconducting quantum bits," Nature, vol. 453, 2008, pp. 1031-1042.

[22] E. Gibney, "Quantum gold rush: the private funding pouring into quantum start-ups," Nature, vol. 574, 2019, pp. 22-24.

[23] G Binnig, H. Rohrer, Ch. Gerber, and E. Weibel, "Tunneling through a controllable vacuum gap," Appl. Phys. Lett., vol. 40, 1982, pp. 178-179.

[24] G. Binnig, H. Rohrer, Ch. Gerber, and E. Weibel, "Surface studies by scanning tunneling microscopy," Phys. Rev. Lett., vol. 49, 1982, pp. 57-60.

[25] G. Binnig, H. Rohrer, Ch. Gerber, and E. Weibel, "7 $\times 7$ Reconstruction on Si (111) resolved in real space," Phys. Rev. Lett., vol. 50, 1982, pp. 120-122.

[26] J. Tersoff and D. R. Hamann, "Theory of the scanning tunneling microscope," Phys. Rev. B, vol. 31, 1985, pp. 805813.

[27] M. Morgenstern, D. Haude, V. Gudmundsson, Chr. Wittneven, R. Dombrowski, Chr. Steinebach, and R. Wiesendanger, "Low temperature scanning tunneling spectroscopy on InAs (110)," Journal of Electron Spectroscopy and Related Phenomena, vol. 109, 2000, pp. 127-145.

[28] L. J. Lauhon and W. Ho, "Direct observation of the quantum tunneling of single hydrogen atoms with a scanning tunneling microscope,” Phys. Rev. Lett., vol. 85, 2000, pp. 4566-4569. 
[29] K. Oura, V. G. Lifshits, A. A. Saranin, A. V. Zotov, and M. Katayama, Surface science: an introduction, Springer-Verlag, 2003, pp. 159-163.

[30] Y.-S. Shin, "The average energy and molar specific heat at constant volume of an Einstein solid measured by an observer with fluctuating frame of reference," Am. J. Phys. Appl., vol. 7, 2019, pp. 21-26.

[31] Y.-S. Shin, "Average current through a single-electron transistor with a channel under fluctuations of an observer's frame of reference," Am. J. Phys. Appl., vol. 7, 2019, pp. 118124.

[32] A. C. Phillips, Introduction to Quantum Mechanics, John Wiley \& Sons Ltd., 2003, pp. 83-108.

[33] R. A. Serway and J. W. Jewett, Physics of Scientists and Engineers with Modern Physics, 9th ed., Brooks/Cole, 2014, p. 1243. 